\title{
A NOTE ON $S$-CLOSED SPACES
}

\author{
ASHA MATHUR ${ }^{1}$
}

\begin{abstract}
A topological space $X$ is said to be $S$-closed if and only if for every semi-open cover of $X$ there exists a finite subfamily such that the union of their closures cover $X$. For a Hausdorff space, the concept of $S$-closed is shown to be equivalent to the concept of extremally disconnected and nearly compact. Further it has been shown that EDH-closed spaces are precisely $S$-closed Hausdorff spaces.
\end{abstract}

T. Thompson introduced and studied $S$-closed spaces in [6]. A space $X$ is said to be $S$-closed if every semi-open cover of $X$ admits a finite subfamily, the closures of whose members cover the space, where a set $S$ is semi-open if and only if there exists an open set $V$ such that $V \subset A \subset \bar{V}$.

Since every regularly closed set is semi-open and the closure of semi-open sets is regularly closed the following result is immediate.

THEOREM 1. The following are equivalent for a topological space $X$ :

(a) $X$ is $S$-closed.

(b) Every regularly closed cover has a finite subcover.

(c) Every family of regularly open sets having the finite intersection property has nonempty intersection.

Since every $S$-closed Hausdorff space is extremally disconnected [6, Theorem 7] and in extremally disconnected spaces regularly closed sets coincide with clopen sets, the following corollary follows from the above theorem.

COROLlARY 2. The following are equivalent for a Hausdorff space $X$ :

(a) $X$ is $S$-closed.

(b) Every regularly closed cover has a finite disjoint clopen refinement.

The main result obtained by $\mathrm{T}$. Thompson is that for compact Hausdorff or compact regular spaces, the concept of $S$-closed is equivalent to the concept of extremally disconnected. Since in a regular space every open cover can be refined by a regularly closed cover it is immediate from Theorem 1(b)

Received by the editors December 15, 1977 and, in revised form, August 19, 1978.

AMS (MOS) subject classifications (1970). Primary 54DXX, 54D20, 54D30; Secondary 54G05. Key words and phrases. $S$-closed, extremally disconnected, nearly compact, EDH-closed.

'The author gratefully acknowledges the financial support given by the Association of Commonwealth Universities by awarding the Commonwealth Scholarship to the author at the University of St. Andrews.

Also, she would like to express her gratitude to Dr. Roy Dyckhoff of the University of St. Andrews for the valuable discussions she had with him during the preparation of this note. 
that a regular $S$-closed space is compact. Thus for regular spaces, Thompson's result gives that the concept of $S$-closed is equivalent to the concept of extremally disconnected and compact. However, the situation is not the same in case of Hausdorff spaces inasmuch as $S$-closed Hausdorff spaces are not necessarily compact. But before we could give an example of such a space we need some more results about $S$-closed spaces. First a couple of definitions.

A space $(X, \mathcal{T})$ is nearly compact [5] if every regularly open cover has a finite subcover and is almost regular [4] if for each point $x$ and a regularly open set $G$ there exists an open set $H$ such that $x \in H \subset \bar{H} \subset G$.

It is immediate from these definitions that in an almost regular space every regularly open cover can be refined by regularly closed sets and hence every almost regular $S$-closed space is nearly compact in view of Theorem 1(b). Using essentially the same proof as that of Theorems 5 and 6 of [6] one can show that every extremally disconnected nearly compact space is $S$-closed and every almost regular $S$-closed space is extremally disconnected. Thus we have:

THEOREM 3. An almost regular space is $S$-closed if and only if it is nearly compact and extremally disconnected.

It may be noted that since almost regular $S$-closed spaces are extremally disconnected, Corollary 2 remains true even for almost regular spaces. Also note that since regular $\Leftrightarrow$ almost regular + semiregular [4] and compact $\Leftrightarrow$ nearly compact + semiregular [5], Thompson's result follows from Theorem 3.

In view of Theorem 7 of [6] and the fact that extremally disconnected spaces are almost regular, the following is immediate from Theorem 3.

THEOREM 4. An Hausdorff space is $S$-closed if and only if it is nearly compact and extremally disconnected.

Robert A. Herrmann [2] constructed a nearly compact Hausdorff extension $\alpha X$ for almost completely regular spaces, where a space $X$ is almost completely regular if for each point $x \in X$ and a regularly closed set $A \boxplus x$ there exists a continuous map $f: X \rightarrow[0,1]$ such that $f(x)=0$ and $f(A)=\{1\}$. It was observed in [2] that $\alpha X$ is compact if and only if $X$ is compact. Further in [1] it has been proved that $\alpha X$ is extremally disconnected if and only if $X$ is extremally disconnected.

Now if $\mathbf{N}$ denotes the set of natural numbers with discrete topology, then $\alpha \mathbf{N}$ is extremally disconnected and hence an $S$-closed space in view of Theorem 4. However, since $\mathbf{N}$ is not compact, $\alpha \mathbf{N}$ is not compact. Thus there exist $S$-closed Hausdorff spaces which are not compact.

However since nearly compact + semiregular $\Rightarrow$ compact [5] we have immediately the following corollary to Theorem 4.

COROLlaRY 5. The following are equivalent for a Hausdorff space $X$ :

(a) $X$ is $S$-closed and semiregular. 
(b) $X$ is compact and extremally disconnected.

(c) $X$ is $S$-closed and regular.

An extremally disconnected Hausdorff space is an EDH-closed space if it is closed in any extremally disconnected Hausdorff space in which it can be embedded. $S$-closed Hausdorff spaces happen to characterize these spaces as shown by the following theorem.

THEOREM 6. The following are equivalent for a space $X$ :

(a) $X$ is $E D H$-closed.

(b) $X$ is $S$-closed and Hausdorff.

(c) $X$ is nearly compact, Hausdorff and extremally disconnected.

(d) $X$ is $H$-closed and extremally disconnected.

Proof. $(a) \Rightarrow(b)$. Since the semiregularization space of an extremally disconnected space is completely regular, every extremally disconnected space is almost completely regular. Thus any given extremally disconnected Hausdorff space $X$ can be densely embedded in a nearly compact Hausdorff space $\alpha H$, which is extremally disconnected since $X$ is extremally disconnected and hence is $S$-closed in view of Theorem 4. Now if $X$ is EDH-closed, then $X$ is a closed subspace of $X$. Since it is already dense in $X$, this implies $X=\alpha X$ and hence $X$ is $S$-closed.

(b) $\Rightarrow$ (c) follows from Theorem 4 and (c) $\Rightarrow$ (d) $\Rightarrow$ (a) are obvious.

It is worth noting that EDH-closed spaces are not necessarily compact or semiregular as $\alpha N$ is an EDH-closed space in view of Theorem 6 but is neither compact nor semiregular. Further it is pointed out that in [3] it was mentioned that (a) and (d) are equivalent and using that equivalence an alternate proof could be provided to Theorem 6 .

\section{REFERENCES}

1. Roy Dyckhoff and Asha Mathur, Nearly compact Hausdorff extensions (unpublished).

2. Robert A. Herrmann, Nearly compact Hausdorff extensions, Glasnik Mat. Ser. III 12 (1977), 125-132.

3. J. R. Porter, Categorical problems in minimal spaces, Lecture Notes in Math., vol. 540, Springer-Verlag, Berlin and New York, 1976, pp. 482-500.

4. M. K. Singal and S. P. Arya, On almost regular spaces, Glasnik Mat. Ser. III 4 (1969), 89-99.

5. M. K. Singal and Asha Mathur, On nearly compact spaces, Boll. Un. Mat. Ital. (4) 6 (1969), 702-710.

6. Travis Thompson, S-closed spaces, Proc. Amer. Math. Soc. 60 (1976), 335-338.

Lady Shri Ram College, Laspat Nagar, New Delhi-110024, India 